University of Nebraska - Lincoln

DigitalCommons@University of Nebraska - Lincoln

\title{
Shear-Horizontal Vibration Modes of an Oblate Elliptical Cylinder and Energy Trapping in Contoured Acoustic Wave Resonators
}

Huijing $\mathrm{He}$

University of Nebraska-Lincoln

Jiashi Yang

University of Nebraska-Lincoln, jyang1@unl.edu

John A. Kosinski

Monmouth University, jkosinsk@monmouth.edu

Follow this and additional works at: https://digitalcommons.unl.edu/mechengfacpub

Part of the Mechanical Engineering Commons

He, Huijing; Yang, Jiashi; and Kosinski, John A., "Shear-Horizontal Vibration Modes of an Oblate Elliptical Cylinder and Energy Trapping in Contoured Acoustic Wave Resonators" (2012). Mechanical \& Materials Engineering Faculty Publications. 74.

https://digitalcommons.unl.edu/mechengfacpub/74

This Article is brought to you for free and open access by the Mechanical \& Materials Engineering, Department of at DigitalCommons@University of Nebraska - Lincoln. It has been accepted for inclusion in Mechanical \& Materials Engineering Faculty Publications by an authorized administrator of DigitalCommons@University of Nebraska Lincoln. 


\title{
Shear-Horizontal Vibration Modes of an Oblate Elliptical Cylinder and Energy Trapping in Contoured Acoustic Wave Resonators
}

\author{
Huijing He, Jiashi Yang, and John A. Kosinski, Fellow, IEEE
}

\begin{abstract}
We study shear-horizontal free vibrations of an elastic cylinder with an oblate elliptical cross section and a traction-free surface. Exact vibration modes and frequencies are obtained. The results show the existence of thickness-shear and thickness-twist modes. The energy-trapping behavior of these modes is examined. Trapped modes are found wherein the vibration energy is largely confined to the central portion of the cross section and little vibration energy is found at the edges. It is also shown that face-shear modes are not allowed in such a cylinder. The results are useful for the understanding of the energy trapping phenomenon in contoured acoustic wave resonators.
\end{abstract}

\section{INTRODUCTION}

Chear vibration modes of crystal plates (including face Oshear, thickness shear, and thickness twist) [1] are the modes used most often for bulk acoustic wave resonators and resonator-based sensors. An important aspect of these modes is their energy trapping behavior. In a partially electroded plate, thickness-shear and thickness-twist vibrations are largely confined to the electroded central region of the plate [2], and fall off rapidly in amplitude outside of this region. Near the edges of the plate there is essentially no vibration and therefore the plate can be mounted at the edges without affecting its vibration. Analysis of the partially electroded plate has shown that the energy trapping is due to mass loading of the elastic plate by the electrodes [2]. Contoured plates with varying thickness (thick in the central region and thin near the edges) can also produce strong energy trapping [3]. Contoured piezoelectric resonators are widely used as electronic components and energy trapping in these resonators has sustained research interest for many years [3]-[15]. However, analyses to date have typically involved approximate solutions, and exact solutions are rare. For example, the analyses in [3]-[7] were based on approximate two-dimensional plate equations for coupled thickness shear and flexure.

Manuscript received February 1, 2012; accepted May 14, 2012. This work was supported in part by the US Army Research Laboratory/US Army Research Office under agreement number W911NF-10-1-0293.

H. He and J. Yang are with the Department of Mechanical and Materials Engineering, University of Nebraska-Lincoln, Lincoln, NE.

J. A. Kosinski is with the Department of Chemistry, Medical Technology, and Physics, Monmouth University, West Long Branch, NJ, and also with the Advanced Technology Group of MacAulay-Brown Inc., Dayton, OH (e-mail: jkosinsk@monmouth.edu).

DOI http://dx.doi.org/10.1109/TUFFC.2012.2381
Alternatively, a single scalar equation for thickness-shear and thickness-twist modes was derived in [8] and [9] for AT- and SC-cut quartz plates, respectively, and this was used in [8]-[12] to analyze contoured resonators. The scalar equation derived in [8], [9] is also two-dimensional and approximate in nature. Mesa resonators with a stepped thickness were studied using combined analytical and numerical (and hence approximate) techniques in [13] for strong energy trapping.

Mathematically, the analysis of contoured resonators leads to differential equations with variable coefficients when the resonators are modeled by two-dimensional equations. This presents considerable mathematical challenges and sometimes additional approximations must be made. Only in rare situations can exact modes be obtained from the three-dimensional equations of elasticity or piezoelectricity. To date, shear-horizontal modes including thickness shear, face shear, and thickness twist in an elastic [14] or a piezoelectric [15] wedge with a linear thickness variation seem to be the only exact solutions available. The solutions for modes in a wedge are useful for understanding the behavior of energy trapping in contoured resonators because a contoured resonator thick in the central region and thin near the edges may be viewed as two wedges joined together (a double wedge according to [3]). In fact, a cylinder with an oblate elliptical cross section (see Fig. 1) may also be viewed as a contoured resonator. It has a smooth surface and is closer to many real contoured resonators than a double wedge. Therefore, in this paper, we study shear-horizontal vibrations in an isotropic elastic cylinder with an oblate elliptical cross section. We have found that this is a rare case among contoured resonators wherein exact modes satisfying the three-dimensional equations of elasticity can be obtained.

\section{Governing EQUATION}

Consider the long cylinder with an elliptical cross-section shown in Fig. 1. The semi-major and semi-minor axes are denoted by $a$ and $b$. We are mainly interested in the case in which $a \gg b$ for contoured resonator application but our solution procedure is valid for any $a$ and $b$ as long as $a \neq b$. For resonator applications, we consider the case in which the surface of the cylinder is traction free. The cylinder is made of an isotropic elastic material. The 


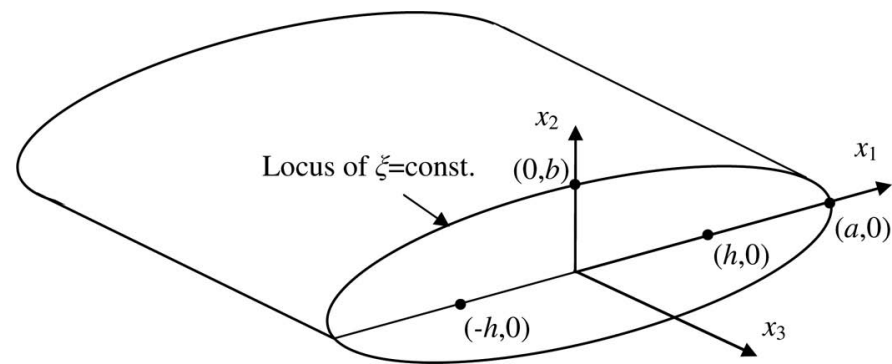

Fig. 1. An oblate elliptical cylinder and coordinate system.

three-dimensional theory of elasticity allows for shear-horizontal motions described by

$$
u_{1}=u_{2}=0, \quad u_{3}=u_{3}\left(x_{1}, x_{2}, t\right),
$$

which depend on time and two spatial variables only. The nonzero components of the strain tensor $S_{i j}$ and the stress tensor $T_{i j}$ are

$$
\begin{aligned}
& S_{4}=2 S_{32}=u_{3,2}, \quad S_{5}=2 S_{31}=u_{3,1}, \\
& T_{4}=\mu u_{3,2}, \quad T_{5}=\mu u_{3,1},
\end{aligned}
$$

where $\mu$ is the shear elastic constant of the material. The nontrivial equation of motion takes the form

$$
c_{2}^{2} \nabla^{2} u_{3}=\ddot{u}_{3},
$$

where $\nabla^{2}$ is the Laplacian operator. $c_{2}$ is the speed of plane shear waves in an infinite isotropic elastic medium, given by

$$
c_{2}^{2}=c_{44} / \rho
$$

We consider time-harmonic free vibrations wherein all fields have the same $\exp (i \omega t)$ factor, which is dropped for simplicity. For such time-harmonic motions, (3) reduces to the Helmholtz equation:

$$
c_{2}^{2} \nabla^{2} u_{3}=-\omega^{2} u_{3}
$$

We introduce elliptical cylindrical coordinates $(\xi, \eta, z)$ defined by [16]

$$
x_{1}=h \cosh \xi \cos \eta, \quad x_{2}=h \sinh \xi \sin \eta, \quad x_{3}=z .
$$

The radial, angular, and axial coordinates $(\xi, \eta, z)$ are over $[0, \infty),[0,2 \pi)$, and $(-\infty, \infty)$ respectively, and curves of constant $\xi$ form ellipses orthogonal to the $x_{3}=z$ axis. The major axis of the cross section of the elliptical cylinder has length $2 a$ along the $x_{1}$ direction and the minor axis has length $2 b$ along the $x_{2}$ direction. The cross section is bounded by an ellipse with foci at $\pm h=\sqrt{a^{2}-b^{2}}$ along the major axis of the cross section. Then, (5) takes the form

$$
\frac{\partial^{2} u_{3}}{\partial \xi^{2}}+\frac{\partial^{2} u_{3}}{\partial \eta^{2}}+2 k^{2}(\cosh 2 \xi-\cos 2 \eta) u_{3}=0,
$$

where

$$
2 k=\frac{\omega}{c_{2}} h .
$$

The nonzero stress components in the elliptic coordinates are

$$
T_{\xi z}=\frac{\mu}{h J} \frac{\partial u_{3}}{\partial \xi}, \quad T_{\eta z}=\frac{\mu}{h J} \frac{\partial u_{3}}{\partial \eta},
$$

where

$$
J=\frac{\sqrt{2}}{2} \sqrt{\cosh 2 \xi-\cos 2 \eta} .
$$

Note that (7)-(10) are dependent only upon the radial and angular elliptic coordinates $(\xi, \eta)$ which describe the cross section of the elliptical cylinder.

\section{Solutions of the Helmholtz Equation}

By the method of separation of variables, we write $u_{3}$ as

$$
u_{3}(\xi, \eta)=U(\xi) V(\eta) .
$$

Substitution of (11) into (7) results in two ordinary differential equations:

$$
\begin{gathered}
\frac{d^{2} V(\eta)}{d \eta^{2}}+(\lambda-2 q \cos (2 \eta)) V(\eta)=0, \\
\frac{d^{2} U(\xi)}{d \xi^{2}}+(2 q \cosh (2 \xi)-\lambda) U(\xi)=0,
\end{gathered}
$$

where $\lambda$ is the separation constant and

$$
q=\frac{\omega^{2} h^{2}}{4 c_{2}^{2}} .
$$

Eqs. (12) and (13) are known as the angular and radial Mathieu equations, respectively.

\section{A. Solutions of the Angular Mathieu Equation}

Eq. (12) has four kinds of periodic solutions with pe$\operatorname{riod} 2 \pi[16]$ :

$$
\begin{gathered}
s e_{2 m+1}(\eta, q)=\sum_{r=0}^{\infty} A_{2 r+1}^{(2 m+1)}(q) \sin (2 r+1) \eta, \\
s e_{2 m+2}(\eta, q)=\sum_{r=0}^{\infty} A_{2 r+2}^{(2 m+2)}(q) \sin (2 r+2) \eta, \\
c e_{2 m}(\eta, q)=\sum_{r=0}^{\infty} B_{2 r}^{(2 m)}(q) \cos (2 r \eta),
\end{gathered}
$$




$$
\left[\begin{array}{ccccccc}
1-\lambda-a & q & 0 & 0 & 0 & 0 & \ldots \\
q & 3^{2}-\lambda & q & 0 & 0 & 0 & \ldots \\
0 & q & 5^{2}-\lambda & q & 0 & 0 & \ldots \\
0 & 0 & q & 7^{2}-\lambda & q & 0 & \ldots \\
\vdots & \vdots & \vdots & \vdots & \vdots & \vdots & \vdots
\end{array}\right]\left[\begin{array}{c}
A_{1}^{(2 m+1)} \\
A_{3}^{(2 m+1)} \\
A_{5}^{(2 m+1)} \\
A_{7}^{(2 m+1)} \\
\vdots
\end{array}\right]=\left[\begin{array}{c}
0 \\
0 \\
0 \\
0 \\
\vdots
\end{array}\right]
$$

$$
\left[\begin{array}{ccccccc}
2^{2}-\lambda & q & 0 & 0 & 0 & 0 & \ldots \\
q & 4^{2}-\lambda & q & 0 & 0 & 0 & \ldots \\
0 & q & 6^{2}-\lambda & q & 0 & 0 & \ldots \\
0 & 0 & q & 8^{2}-\lambda & q & 0 & \ldots \\
\vdots & \vdots & \vdots & \vdots & \vdots & \vdots & \vdots
\end{array}\right]\left[\begin{array}{c}
A_{2}^{(2 m+2)} \\
A_{4}^{(2 m+2)} \\
A_{6}^{(2 m+2)} \\
A_{8}^{(2 m+2)} \\
\vdots
\end{array}\right]=\left[\begin{array}{c}
0 \\
0 \\
0 \\
0 \\
\vdots
\end{array}\right]
$$

$$
\left[\begin{array}{ccccccc}
-\lambda & q & 0 & 0 & 0 & 0 & \ldots \\
2 q & 2^{2}-\lambda & q & 0 & 0 & 0 & \ldots \\
0 & q & 4^{2}-\lambda & q & 0 & 0 & \ldots \\
0 & 0 & q & 6^{2}-\lambda & q & 0 & \ldots \\
\vdots & \vdots & \vdots & \vdots & \vdots & \vdots & \vdots
\end{array}\right]\left[\begin{array}{c}
B_{0}^{(2 m)} \\
B_{2}^{(2 m)} \\
B_{4}^{(2 m)} \\
B_{6}^{(2 m)} \\
\vdots
\end{array}\right]=\left[\begin{array}{c}
0 \\
0 \\
0 \\
0 \\
\vdots
\end{array}\right]
$$

$$
\left[\begin{array}{ccccccc}
1+q-\lambda & q & 0 & 0 & 0 & 0 & \ldots \\
q & 3^{2}-\lambda & q & 0 & 0 & 0 & \ldots \\
0 & q & 5^{2}-\lambda & q & 0 & 0 & \ldots \\
0 & 0 & q & 7^{2}-\lambda & q & 0 & \ldots \\
\vdots & \vdots & \vdots & \vdots & \vdots & \vdots & \vdots
\end{array}\right]\left[\begin{array}{c}
B_{1}^{(2 m+1)} \\
B_{3}^{(2 m+1)} \\
B_{5}^{(2 m+1)} \\
B_{7}^{(2 m+1)} \\
\vdots
\end{array}\right]=\left[\begin{array}{c}
0 \\
0 \\
0 \\
0 \\
\vdots
\end{array}\right]
$$

$$
c e_{2 m+1}(\eta, q)=\sum_{r=0}^{\infty} B_{2 r+1}^{(2 m+1)}(q) \cos (2 r+1) \eta
$$

where $m=0,1,2,3, \ldots$ Eqs. (15)-(18) are solutions of (12) for each $m$. Later, when discussing numerical results, it will become clear that $m$ is associated with the number of nodal lines roughly parallel to the $x_{2}$ axis. $A_{r}^{(m)}(q)$ and $B_{r}^{(m)}(q)$ are coefficients for the series of $\eta$ and they depend on $q$. They are solutions of (19)-(22), see above, obtained by substituting (15)-(18) into (12). $\lambda_{2 m+1}, \lambda_{2 m+2}, \kappa_{2 m}$, and $\kappa_{2 m+1}$ are the roots of the polynomial equations of $\lambda$ obtained by setting the determinants of the coefficient matrices of $(19)-(22)$ to zero. $s e_{2 m+1}(\eta, q)$ and $s e_{2 m+2}(\eta, q)$ correspond to modes antisymmetric about the major axis $\left(x_{1}\right) \cdot c e_{2 m}(\eta, q)$ and $c e_{2 m+1}(\eta, q)$ correspond to symmetric modes. These will become clear later. $\lambda_{2 m+1}$ and $\lambda_{2 m+2}$ are associated with the $(2 m+1)$ th and $(2 m+2)$ th antisymmetric modes, and $\kappa_{2 m}$ and $\kappa_{2 m+1}$ are associated with the $2 m$ th and $(2 m+1)$ th symmetric modes. $c e_{m}(\eta, q)$ and $s e_{m}(\eta, q)$ are orthogonal functions satisfying the condition

$$
\begin{aligned}
\int_{0}^{2 \pi} c e_{m}(\eta, q) c e_{n}(\eta, q) & =\int_{0}^{2 \pi} s e_{m}(\eta, q) s e_{n}(\eta, q) \\
& = \begin{cases}\pi, & \text { if } m=n, \\
0, & \text { if } m \neq n .\end{cases}
\end{aligned}
$$

In accordance with (23), we have the following normalization conditions:

$$
\begin{aligned}
\sum_{r=0}^{\infty}\left(A_{2 r+1}^{(2 m+1)}\right)^{2} & =1, \quad \sum_{r=0}^{\infty}\left(A_{2 r+2}^{(2 m+2)}\right)^{2}=1, \\
2\left(B_{0}^{(2 m)}\right)^{2}+\sum_{r=1}^{\infty}\left(B_{2 r}^{(2 m)}\right)^{2} & =1, \quad \sum_{r=0}^{\infty}\left(B_{2 r+1}^{(2 m+1)}\right)^{2}=1 .
\end{aligned}
$$

\section{B. Solutions of the Radial Mathieu Equation}

The four kinds of radial Mathieu functions corresponding to the angular Mathieu functions are: 


$$
\begin{gathered}
S e_{2 m+1}(\xi, q)=\frac{s e_{2 m+1}^{\prime}(0, q) s e_{2 m+1}\left(\frac{\pi}{2}, q\right)}{\sqrt{q}\left(A_{1}^{(2 m+1)}\right)^{2}} \\
\times \sum_{r=0}^{\infty}(-1)^{m} A_{2 r+1}^{(2 m+1)}\left[J_{m}\left(v_{1}\right) J_{m+1}\left(v_{2}\right)-J_{m}\left(v_{2}\right) J_{m+1}\left(v_{1}\right)\right] \\
S e_{2 m+2}(\xi, q)=-\frac{s e_{2 m+2}^{\prime}(0, q) s e_{2 m+2}^{\prime}\left(\frac{\pi}{2}, q\right)}{q\left(A_{2}^{(2 m+2)}\right)^{2}} \\
\times \sum_{r=0}^{\infty}(-1)^{m} A_{2 r+2}^{(2 m+2)}\left[J_{m}\left(v_{1}\right) J_{m+2}\left(v_{2}\right)-J_{m}\left(v_{2}\right) J_{m+2}\left(v_{1}\right)\right] \\
C e_{2 m}(\xi, q)=\frac{c e_{2 m}(0, q) c e_{2 m}\left(\frac{\pi}{2}, q\right)}{\left(B_{0}^{(2 m)}\right)^{2}} \\
\times \sum_{r=0}^{\infty}(-1)^{m} B_{2 r}^{(2 m)}(q) J_{m}\left(v_{1}\right) J_{m}\left(v_{2}\right), \\
C e_{2 m+1}(\xi, q)=-\frac{c e_{2 m+1}(0, q) c e_{2 m+1}\left(\frac{\pi}{2}, q\right)}{\sqrt{q}\left(B_{1}^{(2 m+1)}\right)^{2}} \\
\times \sum_{r=0}^{\infty}(-1)^{m} B_{2 r+1}^{(2 m+1)}(q)\left[J_{m}\left(v_{1}\right) J_{m+1}\left(v_{2}\right)+J_{m}\left(v_{2}\right) J_{m+1}\left(v_{1}\right)\right]
\end{gathered}
$$

where $v_{1}=\sqrt{q} \exp (-\xi), v_{2}=\sqrt{q} \exp (\xi)$, and $J_{m}$ are Bessel functions of the first kind.

\section{Shear-Horizontal Modes}

We discuss modes antisymmetric and symmetric about the major axis of the oblate elliptical cross section separately.

\section{A. Antisymmetric Modes}

The displacement field of the antisymmetric modes is

$$
u_{3}(\xi, \eta, t)=\sum_{m=1}^{\infty} S_{m} S e_{m}(\xi, q) s e_{m}(\eta, q)
$$

where $S_{m}$ are undetermined constants. Substitution of (29) into (9) gives

$$
\begin{aligned}
& T_{\xi z}=\frac{\mu}{h J} \sum_{m=1}^{\infty} S_{m} S e_{m}^{\prime}(\xi, q) s e_{m}(\eta, q), \\
& T_{\eta z}=\frac{\mu}{h J} \sum_{m=1}^{\infty} S_{m} S e_{m}(\xi, q) s e_{m}^{\prime}(\eta, q),
\end{aligned}
$$

where a prime indicates differentiation with respect to $\xi$ in (30a) or $\eta$ in (30b). Let the elliptical boundary of the cross section of the cylinder be at $\xi=\xi_{0}$. The traction-free boundary condition is

$$
T_{\xi z}=\frac{\mu}{h J} \sum_{m=1}^{\infty} S_{m} S e_{m}^{\prime}\left(\xi_{0}, q\right) s e_{m}(\eta, q)=0,
$$

which implies that

$$
S e_{m}^{\prime}\left(\xi_{0}, q\right)=0
$$

Eq. (32) determines a series of values of $q$ for each $m$. To determine the roots of (32), we plot the left side of (32) versus $q$ to get some estimates of these roots. These estimates are then refined by the bisection method to the desired accuracy. Modes corresponding to different values of $q$ have different numbers of nodal lines roughly parallel to the $x_{1}$ axis. Then $\omega$ can be determined from (14). Note that the case of a fixed boundary with $u_{3}=0$ corresponds to that of a vibrating membrane, for which the free vibration solution was given in [17].

\section{B. Symmetric Modes}

Similarly, the symmetric modes are given by

$$
\begin{gathered}
u_{3}=\sum_{m=0}^{\infty} C_{m} C e_{m}(\xi, q) c e_{m}(\eta, q), \\
T_{\eta z}=\frac{\mu}{h J} \sum_{m=0}^{\infty} C_{m} C e_{m}(\xi, q) c e_{m}^{\prime}(\eta, q), \\
C e_{m}^{\prime}\left(\xi_{0}, q\right)=0 .
\end{gathered}
$$

\section{Numerical Results and Discussion}

As an example, for the elastic material we consider Glass-7950 with $\rho=2180 \mathrm{~kg} / \mathrm{m}^{3}$ and $\mu=2.7815 \times$ $10^{10} \mathrm{~N} / \mathrm{m}^{2}$. The elliptical boundary is oblate with $a=10 \mathrm{~b}$ $=5 \mathrm{~mm}$, which is typical for a resonator. We use the coordinate system definitions from (6) to calculate the radial parameter $\xi_{0}=0.10033$ which describes the surface of the elliptical resonator and the corresponding foci locations at $\pm h= \pm 4.974 \mathrm{~mm}$. For the purposes of this analysis, we use the first thirty terms of each series to calculate the displacement field. The frequency of the most widely

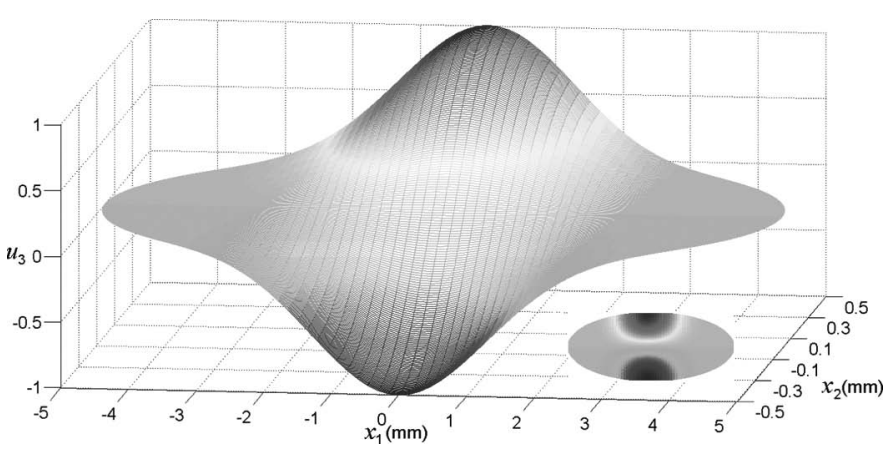

Fig. 2. Fundamental thickness-shear mode. First mode when $u_{3}=$ $S e_{1}(q, \xi) s e_{1}(q, \eta) . \omega=1.1567271 \times 10^{7} \mathrm{rad} / \mathrm{s} . X=1.088 \mathrm{~mm}$. 


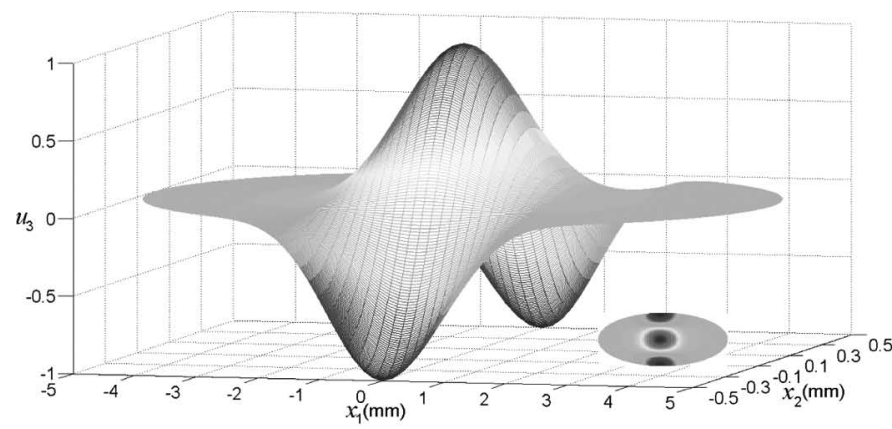

(a)

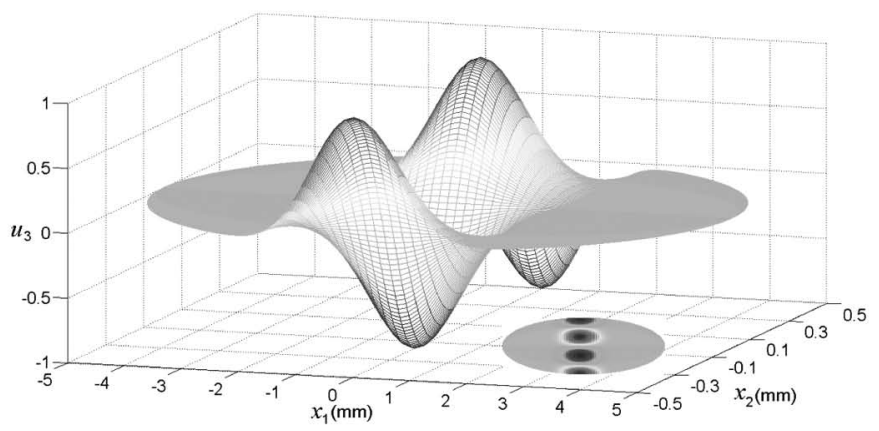

(b)

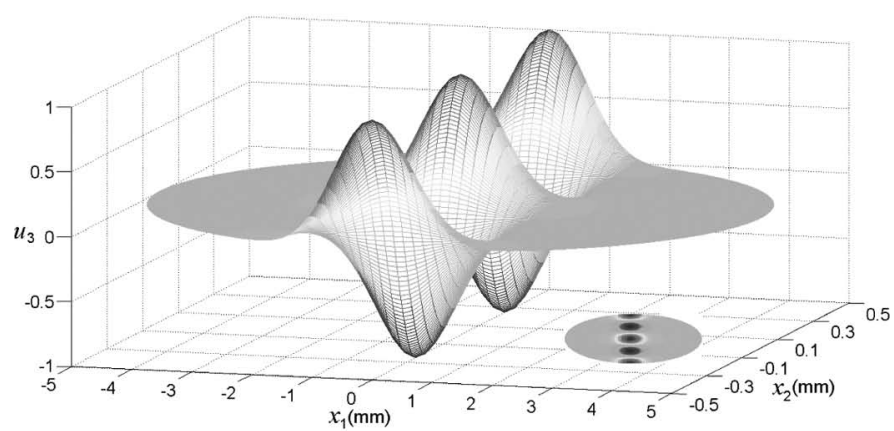

(c)

Fig. 3. Higher-order thickness-shear modes. (a) First mode when $u_{3}=$ $C e_{0}(q, \xi) c \mathrm{e}_{0}(q, \eta) . \omega=2.2795427 \times 10^{7} \mathrm{rad} / \mathrm{s} . X=0.783 \mathrm{~mm}$. (b) Second mode when $u_{3}=S e_{1}(q, \xi) \operatorname{se}_{1}(q, \eta) \cdot \omega=3.4019209 \times 10^{7} \mathrm{rad} / \mathrm{s} . X=$ $0.626 \mathrm{~mm}$. (c) Second mode when $u_{3}=C e_{0}(q, \xi) c e_{0}(q, \eta) \cdot \omega=4.5241962$ $\times 10^{7} \mathrm{rad} / \mathrm{s} . X=0.624 \mathrm{~mm}$.

used fundamental thickness-shear mode is calculated to sixteen significant figures when thirty terms are kept in the series, and this allows for the analysis of phenomenological trends. We recognize that this level of precision far exceeds both the precision of the material constants and the precision of any physical manufacturing processes. Somewhat fewer terms are required to apply the solutions in engineering design. In the figures shown in the following, the maximal displacement is normalized to one. The figures are not drawn to scale. The ellipses are in fact much more oblate than shown in the figures. We will discuss thickness-shear and thickness-twist modes separately.

\section{A. Thickness-Shear Modes}

The fundamental thickness-shear mode is the one most widely used in acoustic wave devices. The displacement

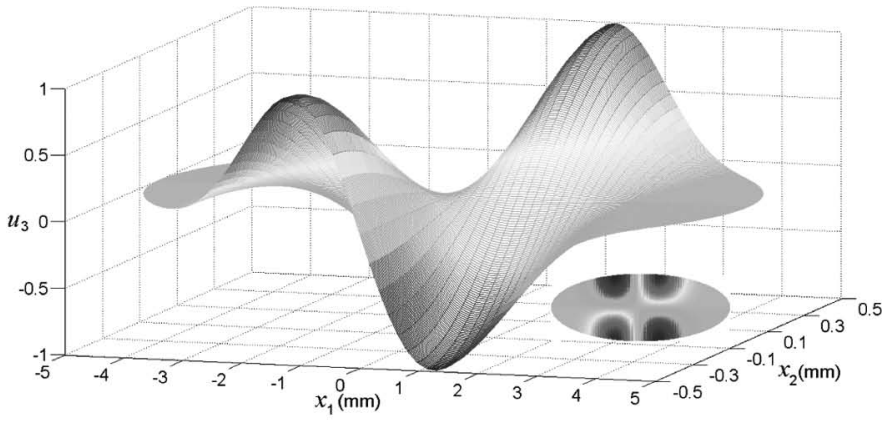

(a)

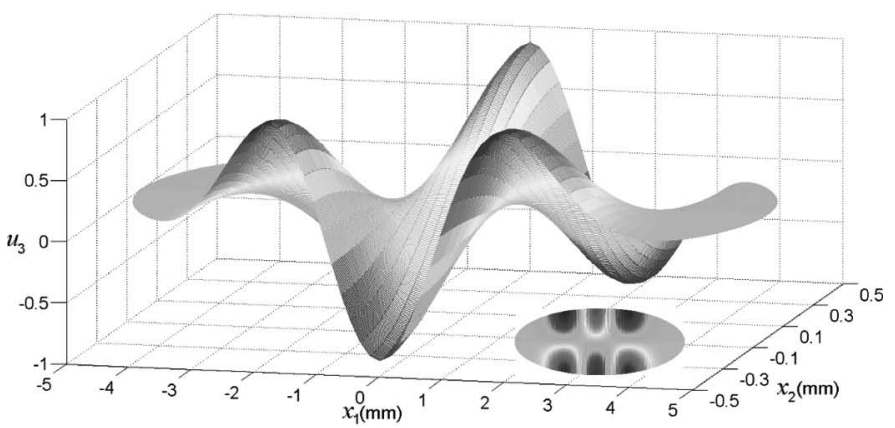

(b)

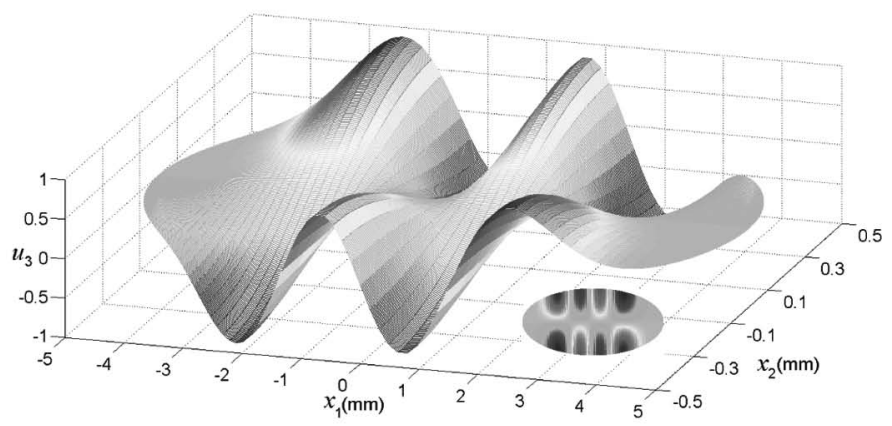

(c)

Fig. 4. Thickness-twist modes. (a) First mode when $u_{3}=S e_{2}(q, \xi)$ $s e_{2}(q, \eta) . \omega=1.2303289 \times 10^{7} \mathrm{rad} / \mathrm{s} . X=1.834 \mathrm{~mm}$. (b) First mode when $u_{3}=S e_{3}(\mathrm{q}, \xi) s e_{3}(q, \eta) . \omega=1.3060256 \times 10^{7} \mathrm{rad} / \mathrm{s} . X=2.263 \mathrm{~mm}$. (c) First mode when $u_{3}=S e_{4}(q, \xi) s e_{4}(q, \eta) \cdot \omega=1.3837045 \times 10^{7} \mathrm{rad} / \mathrm{s}$. $X=2.543 \mathrm{~mm}$.

distribution of this mode over a cross section is shown in Fig. 2. The inset shows the cross section; darker areas represent larger displacements. This mode has one nodal line with zero displacement at $x_{2}=0$ or along the $x_{1}$ axis. When the upper half of the cross section is moving in one direction, the lower half moves in the opposite direction or vice versa. We note that although the top and bottom of the cylinder are moving with the largest displacement, the left and right edges are not moving. This is the so-called energy trapping phenomenon. With energy trapping, the cylinder can be mounted at the left and/or right edges without affecting the vibration in the central region. To quantify energy trapping, we introduce a characteristic $X$ which is determined as the central portion of the cross section with $\left|x_{1}\right|<X$ which carries $90 \%$ of the vibration energy. If we fix $b$ and increase $a$, when $a / b$ is very large, 


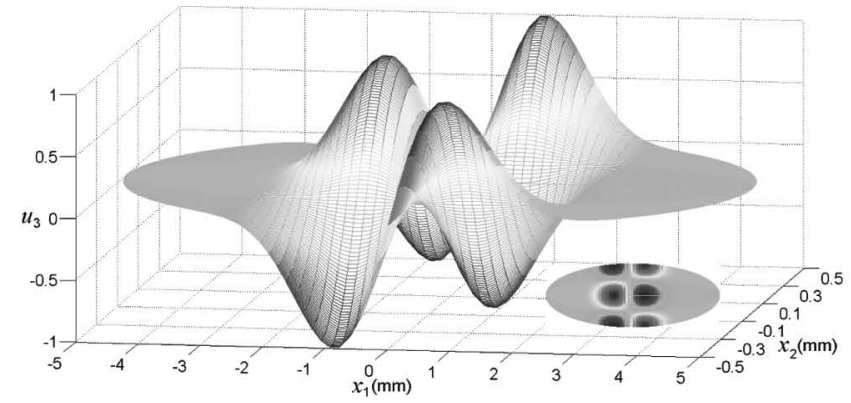

(a)

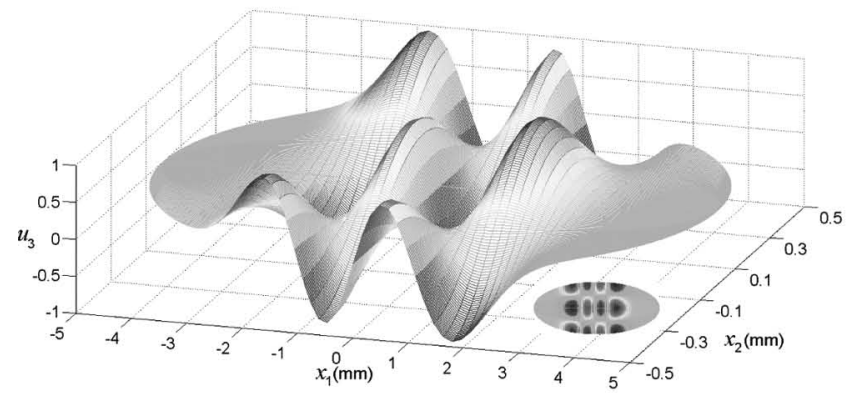

(c)

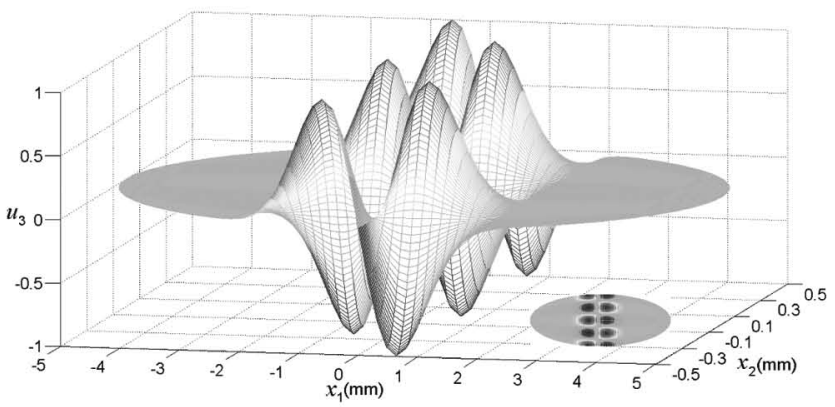

(e)

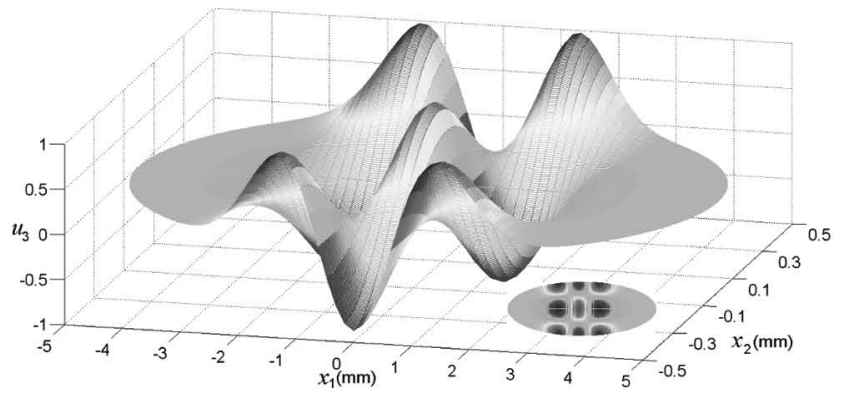

(b)

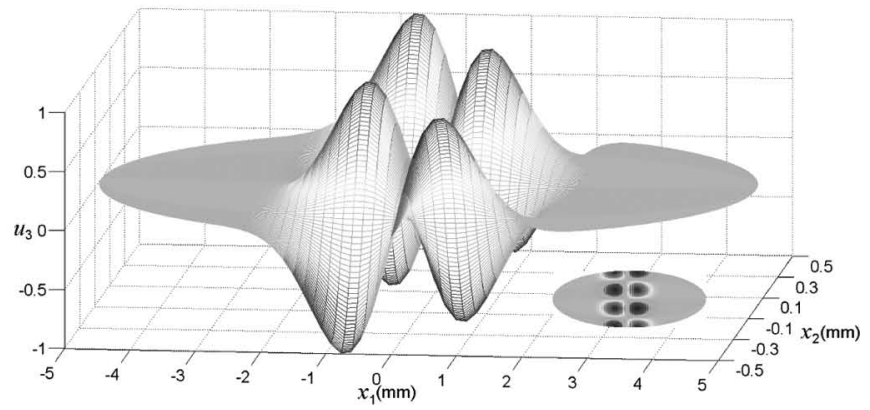

(d)

Fig. 5. Other thickness-twist modes. (a) First mode when $u_{3}=C e_{1}(q, \xi) c e_{1}(q, \eta) . \omega=2.3521836 \times 10^{7} \mathrm{rad} / \mathrm{s} . X=1.243 \mathrm{~mm}$. (b) First mode when $u_{3}=C e_{2}(q, \xi) c e_{2}(q, \eta) \cdot \omega=2.4259184 \times 10^{7} \mathrm{rad} / \mathrm{s} . X=1.686 \mathrm{~mm}$. (c) First mode when $u_{3}=C e_{3}(q, \xi) c e_{3}(q, \eta) \cdot \omega=2.5007192 \times 10^{7} \mathrm{rad} / \mathrm{s} . X=$ $1.976 \mathrm{~mm}$. (d) Second mode when $u_{3}=S e_{2}(q, \xi) s e_{2}(q, \eta) . \omega=3.4742139 \times 10^{7} \mathrm{rad} / \mathrm{s} . X=1.086 \mathrm{~mm}$. (e) Second mode when $u_{3}=C e_{1}(q, \xi) c e_{1}(q, \eta) . \omega$ $=4.5963106 \times 10^{7} \mathrm{rad} / \mathrm{s} . X=0.934 \mathrm{~mm}$.

we expect the result to approach that of a narrow rectangular cross section. Our numerical tests show that when $a / b=10,20,30$, and 40 , the frequency of the fundamental thickness-shear mode is $1.1567,1.1397,1.1340$, and 1.1310 $\times 10^{7} \mathrm{~Hz}$, respectively, approaching $1.1222 \times 10^{7} \mathrm{~Hz}$ of a rectangular cross section from above, as expected. The fundamental thickness-shear mode of a rectangular cross section is $x_{1}$ - or $a$-independent. Our solution cannot be directly used to calculate the special case of a circular cylinder with $a=b$ because in this case $h=q=0$. Instead, we calculate the case of an almost circular cylinder with $a$ $=5 \mathrm{~mm}$ and $b=0.99 a$ whose first fundamental thicknessshear frequency is found to be $1.3281 \times 10^{6} \mathrm{~Hz}$, very close to that of a circular cylinder with a radius of $5 \mathrm{~mm}$ which is $1.3153 \times 10^{6} \mathrm{~Hz}$ [18].

Figs. 3(a), 3(b), and 3(c) show three higher-order thickness-shear modes. They have 2, 3, and 4 nodal lines roughly parallel to the $x_{1}$ axis, respectively. The frequencies of the thickness-shear modes in Fig. 2 and Figs. 3(a), $3(\mathrm{~b})$, and 3(c) are roughly equally spaced, but not exactly so. The modes in Fig. 2 and Fig. 3(b) are antisymmetric about the $x_{1}$ axis. The other two modes are symmetric. We note that higher-order modes are better trapped, i.e., with narrower vibration distributions in the $x_{1}$ direction.

\section{B. Thickness-Twist Modes}

Corresponding to the fundamental thickness-shear mode in Fig. 2 with a nodal line at $x_{2}=0$, there are higher-order modes with additional roughly vertical nodal lines, as shown in Figs. 4(a), 4(b), and 4(c) with an increasing number of 1,2 , and 3 vertical nodal lines, respectively. These are called thickness-twist modes. We note that the distribution of the modes in Figs. 4(a), 4(b), and $4(\mathrm{c})$ in the $x_{1}$ direction increases for higher-order modes, indicating that the higher-order modes are trapped less. Unlike the nearly harmonic frequencies of the thicknessshear modes, the frequencies of the thickness-twist modes in Fig. 4(a), 4(b), and 4(c) increase only slightly for the higher-order modes. 
Similarly, Fig. 5(a), 5(b), and 5(c) show three thicknesstwist modes corresponding to the higher-order thicknessshear mode in Fig. 3(a). Figs. 5(d) and 5(e) are thicknesstwist modes corresponding to the thickness-shear modes in Figs. 3(b) and 3(c), respectively.

\section{CONCLUSION}

Exact solutions for shear-horizontal modes have been obtained for an oblate elastic elliptical cylinder. These modes show energy trapping. Higher-order thicknessshear modes exhibit rapidly increasing (nearly harmonic) frequencies and higher-order modes are increasingly better trapped, as shown in Fig. 3. In contrast, higher-order thickness-twist modes exhibit slowly increasing frequencies and the higher-order modes are increasingly less trapped, as shown in Fig. 4. The exact mode solutions obtained are rare and provide basic understandings of energy trapping in contoured acoustic wave resonators. Analysis of elliptical cylinders made from anisotropic and/or piezoelectric materials can provide further understanding of contoured resonators but is mathematically more challenging.

\section{REFERENCES}

[1] R. D. Mindlin, An Introduction to the Mathematical Theory of Vibrations of Elastic Plates. J. S. Yang, Ed., Singapore: World Scientific, 2006.

[2] R. D. Mindlin and P. C. Y. Lee, "Thickness-shear and flexural vibrations of partially plated, crystal plates," Int. J. Solids Struct., vol. 2, no. 1, pp. 125-139, 1966.

[3] R. D. Mindlin and M. Forray, "Thickness-shear and flexural vibrations of contoured crystal plates," J. Appl. Phys., vol. 25, no. 1, pp. $12-20,1954$.

[4] J. Bleustein, "Thickness-twist and face-shear vibrations of a contoured crystal plate," Int. J. Solids Struct., vol. 2, no. 3, pp. 351-360, 1966.

[5] P. C. Y. Lee and S.-S. Chen, "Vibrations of contoured and partially plated, contoured, rectangular, AT-cut quartz plates," J. Acoust. Soc. Am., vol. 46, no. 5B, pp. 1193-1202, 1969.

[6] P. C. Y. Lee and J. Wang, "Piezoelectrically forced thickness-shear and flexural vibrations of contoured quartz resonators," J. Appl. Phys., vol. 79, no. 7, pp. 3411-3422, 1996.

[7] J. Wang, "Thickness-shear and flexural vibrations of linearly contoured crystal strips with multiprecision computation," Comput. Struc., vol. 70, no. 4, pp. 437-445, 1999.

[8] H. F. Tiersten and R. C. Smythe, "An analysis of contoured crystal resonators operating in overtones of coupled thickness shear and thickness twist," J. Acoust. Soc. Am., vol. 65, no. 6, pp. 1455-1460, 1979.

[9] D. S. Stevens and H. F. Tiersten, "An analysis of doubly rotated quartz resonators utilizing essentially thickness modes with transverse variation," J. Acoust. Soc. Am., vol. 79, no. 6, pp. 1811-1826, 1986 .

[10] H. F. Tiersten, B. J. Lwo, and B. Dulmet, J. Appl. Phys., vol. 80, no. 2 , pp. 1037-1046, 1996.

[11] H. F. Tiersten and Y. S. Zhou, "Transversely varying thickness modes in quartz resonators with beveled cylindrical edges," J. Appl. Phys., vol. 76, no. 11, pp. 7201-7208, 1994.

[12] J. S. Yang, "An analysis of partially electroded, contoured quartz resonators with beveled cylindrical edges," IEEE Trans. Ultrason. Ferroelectr. Freq. Control, vol. 54, no. 11, pp. 2407-2409, 2007.

[13] H. Sekimoto, S. Goka, and Y. Watanabe, "Analysis of 3-D vibrations of rectangular AT-cut quartz plates with bi-mesa structure," IEEE Trans. Ultrason. Ferroelectr. Freq. Control, vol. 48, no. 5, pp. 1302-1307, 2001.

[14] J. S. Yang and H. G. Zhou, "Vibrations of an isotropic elastic wedge," IEEE Trans. Ultrason. Ferroelectr. Freq. Control, vol. 52, no. 1, pp. 139-144, 2005.
[15] J. S. Yang, "Shear horizontal vibrations of a piezoelectric/ferroelectric wedge," J. Acta Mechanica, vol. 173, no. 1-4, pp. 13-17, 2004.

[16] N. W. McLachlan, Theory and Application of Mathieu Functions. Oxford, UK: Oxford Press, 1951.

[17] J. Gutiérrez-Vega, S. Chávez-Cerda, and R. Rodríguez-Dagnino, "Free oscillations in an elliptic membrane," Rev. Mex. Fis., vol. 45, no. 6, pp. 613-622, 1999.

[18] J. H. Baltrukonis and W. G. Gottenberg, "Thickness-shear vibration of circular bars," J. Acoust. Soc. Am., vol. 31, no. 6, pp. 734-739, 1959.

Huijing He's photograph and biography were unavailable at time of publication.

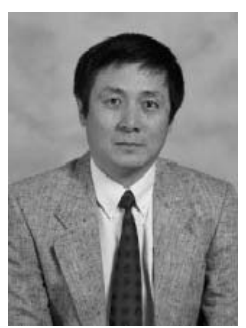

Jiashi Yang received his B.E. and M.E. degrees in engineering mechanics in 1982 and 1985 from Tsinghua University, Beijing, China, and his $\mathrm{Ph} . \mathrm{D}$. degree in civil engineering in 1994 from Princeton University, Princeton, NJ. He was a Postdoctoral Fellow from 1993 through 1994 at the University of Missouri-Rolla, Rolla, MO, and from 1994 through 1995 at Rensselaer Polytechnic Institute, Troy, NY. He was employed by Motorola, Inc., Schaumburg, IL, from 1995 through 1997.

Since 1997 he has been an Assistant and Associate Professor at the Department of Engineering Mechanics of the University of Nebraska-Lincoln, Lincoln, NE. Dr. Yang has published over 170 journal papers on electromechanical materials and devices, and three books: An Introduction to the Theory of Piezoelectricity, The Mechanics of Piezoelectric Structures, and Analysis of Piezoelectric Devices.

John A. Kosinski (A'86-M'89-SM'91-F'03) received the A.A. degree in science in 1978 from Ocean County College, Toms River, NJ, the B.S. degree in physics in 1980 from Montclair State College, Upper Montclair, NJ, the M.S. degree in electronic engineering in 1988 from Monmouth College, West Long Branch, NJ, and the Ph.D. degree in electrical engineering in 1993 from Rutgers, the State University of New Jersey, Piscataway, NJ. He currently teaches physics, astronomy, and integrated science as an Adjunct Professor at Monmouth University, West Long Branch, NJ, while also working as a Senior Consulting Scientist for the Advanced Technology Group of MacAulay-Brown, Inc., Dayton, OH.

From 1981 to 2011, Dr. Kosinski served as a civilian employee of the U.S. Army at Fort Monmouth, NJ, first at the U.S. Army Electronics Technology and Devices Laboratory, and subsequently at the U.S. Army Communications-Electronics Research, Development, and Engineering Center (CERDEC). There he enjoyed a broad research career touching upon many areas of interest to the IEEE, including the development and characterization of new piezoelectric materials, design of electronic components and modules, novel approaches to electromagnetic interference (EMI) suppression, and sensor, signal, and information processing. Dr. Kosinski received the Department of the Army Research and Development Achievement Award six times for work in the areas of SAW device acceleration sensitivity, electro-diffusion processing of quartz material, high-power microwave EMI protection, understanding of the fundamental nature of acceleration sensitivity in phase-coherent signal sources, the development of electronics for tactical unmanned aerial vehicles, and the application of information theory to a military identification problem. Perhaps most significantly, he received the Superior Civilian Service Award for his role in a specially equipped team deployed to the World Trade Center to assist in victim rescue and evidence recovery in response to the terrorist attacks of September 11, 2001.

Dr. Kosinski has published more than 150 technical articles, including numerous invited papers and tutorials, and holds 27 U.S. patents. He has been an elected member of the IEEE Ultrasonics, Ferroelectrics, and Frequency Control (UFFC) Society Administrative Committee (AD$\mathrm{COM}$ ) and an Associate Editor of the IEEE Transactions on Ultrasonics, Ferroelectrics, and Frequency Control. In the past, he has served on the technical program committees of the IEEE International Frequency Control Symposium and the International Ultrasonics Symposium, including serving as General Chair for the latter Symposium in 2007. He is a Senior Research Fellow of and former Director of Admissions for the International Society for Philosophical Enquiry (ISPE), a global nonprofit organization dedicated to the advancement of human knowledge through personal accomplishment, advanced enquiry, and creative contributions. 